PROCEEDINGS of the Tenth International Driving Symposium on Human Factors in Driver Assessment, Training and Vehicle Design

\title{
THE EFFECT OF TURN SIGNAL ONSET ON LATERAL PERFORMANCE MEASURES WHEN OVERTAKING A LEAD VEHICLE - USING NATURALISTIC DRIVING ENVIRONMENT
}

\author{
Brian T. W. Lin ${ }^{1}$, Shan Bao ${ }^{1,2}$ \\ ${ }^{1}$ University of Michigan Transportation Research Institute \\ Ann Arbor, MI, USA \\ ${ }^{2}$ Industrial and Manufacturing Systems Engineering, University of Michigan-Dearborn \\ Dearborn, MI, USA \\ Email: btwlin@umich.edu
}

\begin{abstract}
Summary: Lane changes occur very frequently on freeways. For the development of automated vehicles (AV), the detection of the other vehicles' lane change maneuvers is an important task. Practically, turn signal is the most direct indicator to show the driver's intention to change lanes. This study explored the Safety Pilot field-operational-test (SPFOT) database to investigate the use of turn signal and the relationship between the turn signal onset time and lane change performance measures, in order to assist AV anticipating other road users' maneuvers. Driving data from 130 instrumented vehicles were extracted and 31,211 overtaking events were selected. It was found that the turn signal was used for about $70 \%$ of lane changes, and during half of those the turn signal was activated after the initiation of the lane change maneuver. Results showed that leftward overtaking maneuvers had longer lane change duration with slower lateral speed and lateral acceleration than rightward ones when the turn signal was not used. It was further found that the lane change duration can be estimated by the turn signal onset time. The shortest lane change durations of $5.33 \mathrm{~s}$ and $4.66 \mathrm{~s}$ occurred during those maneuvers when the turn signal was activated at $4.5 \mathrm{~s}$ and $5 \mathrm{~s}$ before the start of the leftward and rightward lane changes, respectively.
\end{abstract}

\section{INTRODUCTION}

On freeways, drivers change lanes frequently for many reasons, such as overtaking, taking the exit ramp, entering the freeway, staying away from obstacles, and returning to the original lane after overtaking. Due to this frequent maneuver, the design of automated vehicles (AV) need to detect the intention and maneuver of these lane changes of both ego and surrounding vehicles. Previous research have developed different mathematical models to predict lane change intentions by using steering wheel angle and yaw rate (Schmidt et al., 2014), lateral position (Kumar et al., 2013), cooperative strategy (Wang et al., 2015), or eye glance and head motion (Doshi \& Trivedi, 2008). To obtain these input data for the AV, however, vehicle dynamic is greatly influenced by road geometry and the environment, lateral position needs accurate detection of lane markers, cooperative driving strategy changes a lot in complicated traffic conditions, and eye gazes of the other road users are difficult to detect. Many limitations in the models had been found.

From our perspectives, turn signal is a more straight-forward indicator that notifies the AV with the upcoming maneuver of the other vehicles, and it can be recognized accurately (Fröhlich et al., 2014) even in the nighttime (Chen et al., 2014). At the onset of turn signal activation, drivers 
clearly indicate the intention of a lane change and decides how this lane change will be executed in a short period of time. Therefore, it is critical to understand how the turn signal use are represented in driver's decision in terms of lane change intention and maneuvers.

\section{OBJECTIVES}

This research investigated the use of turn signal and aimed to connect the turn signal onset time with drivers' lane change maneuvers under different conditions. This was done by exploring exiting data from a naturalistic driving environment. This study focuses on the lane change maneuver for overtaking purposes, which has been reported as the main reason for vehicles changing lanes (Lee et al., 2004). The research questions for this study are as follows:

(1) How frequently will a driver activate the turn signal when changing lanes?

(2) For those who engage the turn signal when changing lanes, when is the turn signal onset time during lane change profile?

(3) How do the lane change maneuvers differ based on the use of turn signal?

(4) How does the turn signal onset time correlate with the lane change maneuver profile?

\section{METHOD}

\section{Data preparation}

This research is mining the Safety Pilot field-operational-test (SPFOT, Bezzina \& Sayer, 2015) database to explore drivers' lane change maneuvers. In SPFOT, 130 participants' cars were instrumented with data acquisition systems (DAS), so the vehicle dynamic data including speed, acceleration, throttle position, turn signal engagement, etc., with the video showing the views in the cabin, were collected in the frequency of $10 \mathrm{~Hz}$. These instrumented vehicles were equipped with Mobileye camera that was able to detect the object in the front (usually lead vehicles) and its range and range rate to the instrumented vehicle, as well as the lane boundary that can be used to estimate the lane position. During the time period the DAS installed, these participants used their cars as usual in their daily lives.

Only drivers who participated in SPFOT for longer than one year would be used for data extraction in this study and only the data collected in the first year would be used. Qualified lane changes are defined to satisfy four conditions:

(1) the driving speed was greater than $55 \mathrm{mph}$,

(2) there was a lead vehicle in the ego lane when the lane change began,

(3) the lead vehicle in the ego lane was passed by the ego vehicle after the completion of the lane change

(4) the lane change was completed without aborting.

For each lane change event, the starting point was defined as when the vehicle just started to move towards the target lane (no reverse movement afterwards), and ending point was when the vehicle fully presented in the target lane and started to move toward the opposite direction (minor adjustment). Figure 1 shows an example for the starting and ending points of a single lane change towards the left. The data extraction window was from $10 \mathrm{~s}$ before the starting point to the ending point. The $10 \mathrm{~s}$ window helps understand what the driver was doing before starting a lane change. 


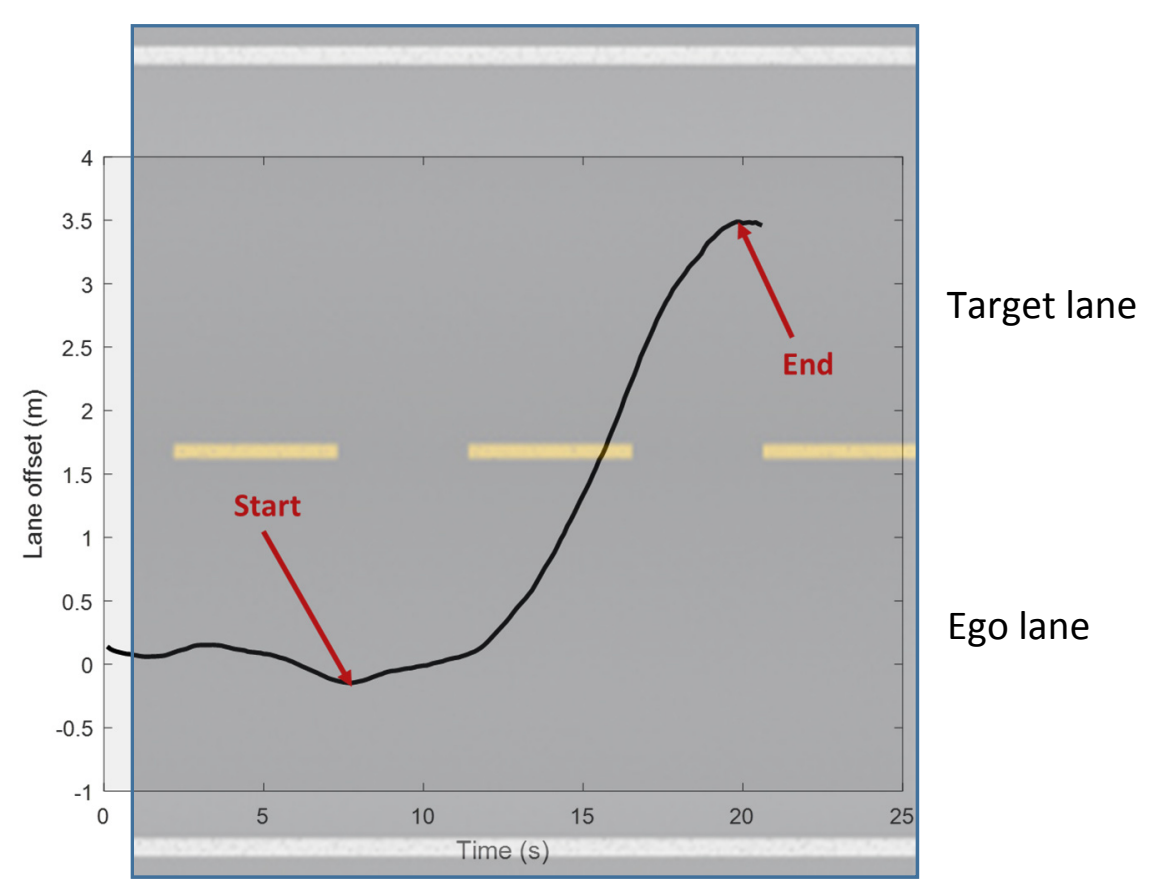

Figure 1. Example: Lane offset (lateral) trajectory when changing lane to the left

\section{Variables}

The independent variables included the engagement of turn signal and lane change direction. The dependent variables included the lateral speed and lateral acceleration, which were calculated as the mean and maximum values within the time windows of "starting and ending points". In addition, lane change duration (how long a lane change took) was selected since it was highly correlated with the lane change trajectory (Tijerina \& Hetrick, 1997).

\section{RESULTS}

\section{Turn signal onset time}

With the above-mentioned method, 31,211 lane change events were selected from the SPFOT database. Table 1 shows the counts of lane changes with turn signal engaged at different time point during lane changes. With the $10 \mathrm{~s}$ data extraction window, it was found that $3 \%$ of lane changes had turn signals activated at $10 \mathrm{~s}$ before starting a lane change or earlier. These cases would be filtered out. The turn signal engagement for leftward and rightward lane changes did not differ much. For about $30 \%$ of lane changes, the turn signal was not used at all within the data collection window of each lane change event. The proportions of activating the turn signal before and after the "start of lane changes" were similar (32-35\%), for both leftward and rightward lane changes.

Table 1. Turn signal (TS) onset time

\begin{tabular}{|l|r|r|r|r|}
\hline \multirow{2}{*}{} & \multicolumn{2}{|c|}{ Left (16,299 lane changes) } & \multicolumn{2}{|c|}{ Right (14,912 lane changes) } \\
\cline { 2 - 2 } & TS engaged & TS not engaged & TS engaged & TS not engaged \\
\hline Before LC starts & $5,640(35 \%)$ & & $4,699(32 \%)$ & \multirow{2}{*}{$4,428(30 \%)$} \\
\cline { 1 - 2 } 10 s before LC starts & $453(3 \%)$ & $516(28 \%)$ & $5 \%(3 \%)$ & \\
\cline { 1 - 2 } & & $5,613(34 \%)$ & $5,269(35 \%)$ & \\
\hline
\end{tabular}


However, the turn signal onset time before and after the lane change distributes differently as lane change direction, as shown in Figure 2. Drivers tend to activate the turn signal right before or after when they start a lane change. For those who activated the turn signal before the lane change, the turn signal onset time for rightward lane changes was $0.5 \mathrm{~s}$ earlier than leftward lane changes. For the cases of activating the turn signal after the lane change, the onset time for rightward lane changes was $0.23 \mathrm{~s}$ later than the leftward ones.

Leftward lane changes
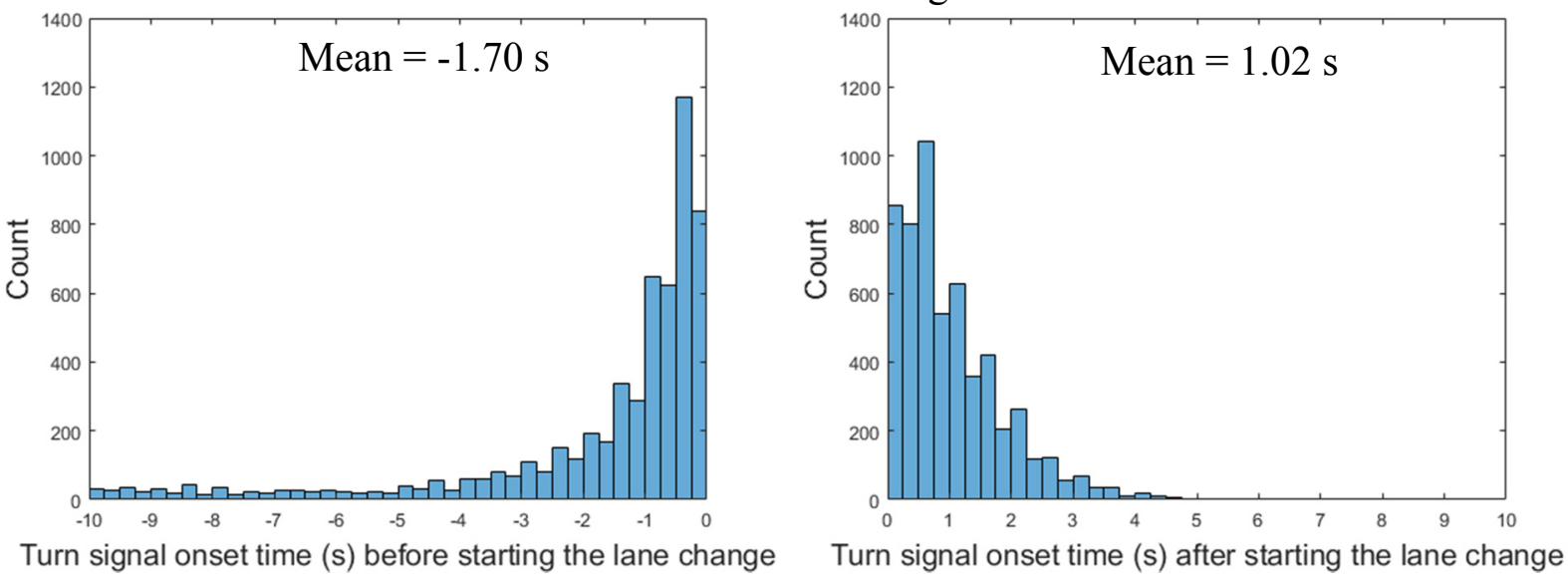

Rightward lane changes
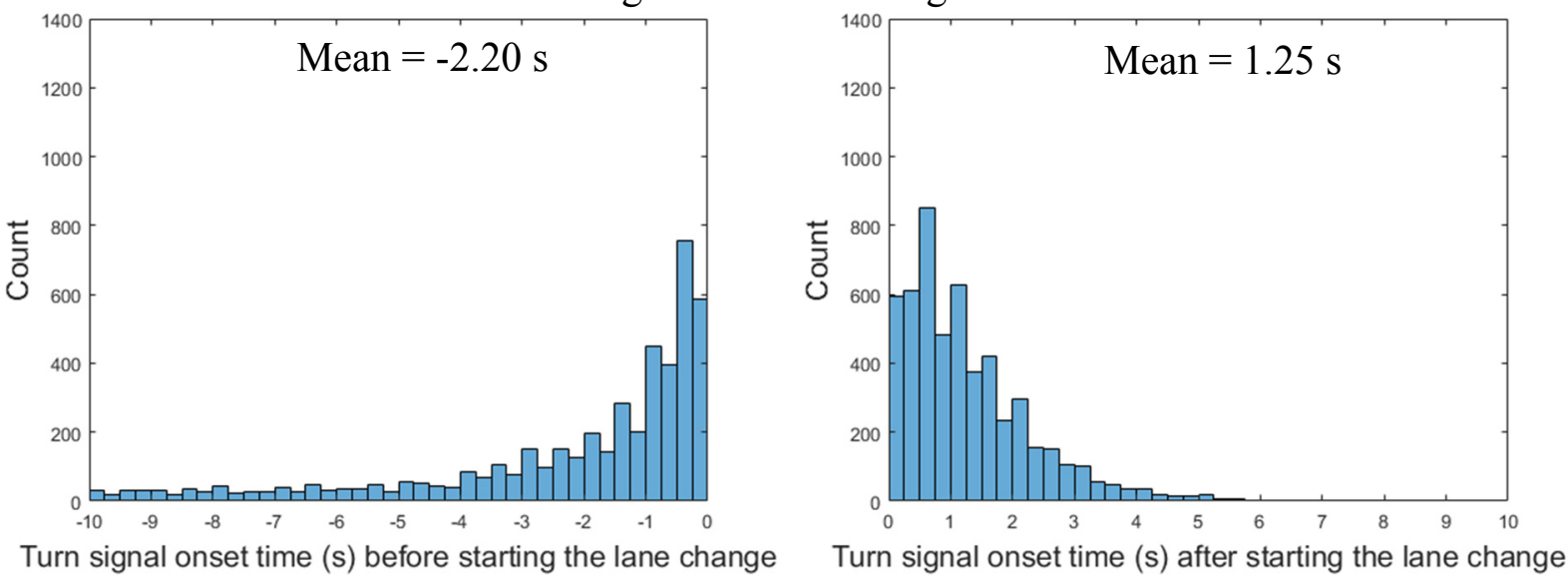

Figure 2. Distributions of turn signal onset time for leftward and rightward lane changes

\section{Effects of turn signal engagement and lane change direction on lane change maneuvers}

Two-way ANOVA was conducted to find the effect of turn signal engagement and lane change direction on lane change performance measures, as shown in Table 2. Lane change direction had significant effect on most lane change performance measures that drivers had longer lane change duration, smaller lateral speed and smaller lateral acceleration. The effect of turn signal engagement was significant on lane change duration and mean lateral acceleration. When the turn signal was engaged, the lane change duration was greater, with smaller lateral acceleration. Neither lane change direction nor the turn signal engagement significantly affect the maximum lateral acceleration. 
Lane change direction had significant interaction with turn signal engagement on all the performance measures except the maximum lateral acceleration. When changing lane with the turn signal engaged, the performance difference between leftward and rightward lane changes was always smaller than the ones without engaging the turn signal. Therefore, once drivers activated the turn signal to indicate their intention to change lane, the performance for leftward and rightward lane changes was similar with smaller variations. Otherwise, the rightward lane changes would be executed with shorter duration, greater lateral speed and acceleration.

\section{Estimating lane change maneuver using turn signal onset time}

Only performance measures with significant effect of turn signal engagement were selected for prediction modeling, so only the lane change duration and mean lateral acceleration were included. Furthermore, since the change of lateral speed was a continuous process, averaging the lateral acceleration could over-simplify the measure and prediction model. Therefore, in this study we would only model the lane change duration. Second degree polynomial models were used to fit since the effects of turn signal onset times before and after the lane change might be opposite. Table 3 shows the prediction for these performance measures. The prediction for lane change duration indicated a minimum lane change duration when the turn signal onset time was about $4.5 \mathrm{~s}$ and $5 \mathrm{~s}$ before the start of a leftward and rightward lane change, respectively (see Figure 3). The result was consistent to what was found that the right turn signal onset time was $0.5 \mathrm{~s}$ earlier than leftward. When turn signal was activated at these two timings, drivers tend to move faster towards the target lane.

Table 2. Lane change (LC) performance measures by the engagement of the turn signal (TS) and lane change direction

\begin{tabular}{|c|c|c|c|c|c|c|}
\hline \multicolumn{4}{|c|}{ Statistic } & \multicolumn{3}{|c|}{ ANOVA } \\
\hline Performance measure & $\begin{array}{c}\mathrm{LC} \\
\text { direction }\end{array}$ & $\begin{array}{c}\text { TS } \\
\text { engaged }\end{array}$ & $\begin{array}{c}\text { TS not } \\
\text { engaged }\end{array}$ & Source & $\mathbf{F}$ & $\mathbf{p}$ \\
\hline \multirow{3}{*}{ LC duration (s) } & Left & 6.6593 & 6.7954 & LC direction & 196.71 & $<0.001$ \\
\hline & Right & 6.4802 & 6.1608 & TS engagement & 9.97 & 0.002 \\
\hline & & & & $\mathrm{LC} \times \mathrm{TS}$ & 61.63 & $<0.001$ \\
\hline \multirow{3}{*}{$\begin{array}{l}\text { Mean lateral speed } \\
(\mathrm{m} / \mathrm{s})\end{array}$} & Left & 0.4911 & 0.4766 & LC direction & 27.07 & $<0.001$ \\
\hline & Right & 0.4911 & 0.5220 & TS engagement & 3.56 & 0.06 \\
\hline & & & & $\mathrm{LC} \times \mathrm{TS}$ & 27.09 & $<0.001$ \\
\hline \multirow{3}{*}{$\begin{array}{l}\text { Maximum lateral speed } \\
(\mathrm{m} / \mathrm{s})\end{array}$} & Left & 1.1040 & 1.0349 & LC direction & 9.47 & 0.002 \\
\hline & Right & 1.0683 & 1.2096 & TS engagement & 2.56 & 0.11 \\
\hline & & & & $\mathrm{LC} \times \mathrm{TS}$ & 21.7 & $<0.001$ \\
\hline \multirow{3}{*}{$\begin{array}{l}\text { Mean lateral } \\
\text { acceleration }\left(\mathrm{m} / \mathrm{s}^{2}\right)\end{array}$} & Left & 0.0080 & 0.0120 & LC direction & 72.64 & $<0.001$ \\
\hline & Right & 0.0144 & 0.0298 & TS engagement & 46.31 & $<0.001$ \\
\hline & & & & $\mathrm{LC} \times \mathrm{TS}$ & 16.06 & $<0.001$ \\
\hline \multirow{3}{*}{$\begin{array}{l}\text { Maximum lateral } \\
\text { acceleration }\left(\mathrm{m} / \mathrm{s}^{2}\right)\end{array}$} & Left & 0.1046 & 0.0837 & LC direction & 0.07 & 0.80 \\
\hline & Right & 0.0894 & 0.0935 & TS engagement & 0.62 & 0.43 \\
\hline & & & & $\mathrm{LC} \times \mathrm{TS}$ & 1.37 & 0.24 \\
\hline
\end{tabular}


Table 3. Prediction for lane change duration (Y) with turn signal onset time (X)

\begin{tabular}{|c|c|c|}
\hline Y & Leftward & Rightward \\
\hline Lane change duration (s) & $0.0620 x^{2}+0.556 x+6.577$ & $0.0682 x^{2}+0.6709 x+6.31$ \\
\hline
\end{tabular}
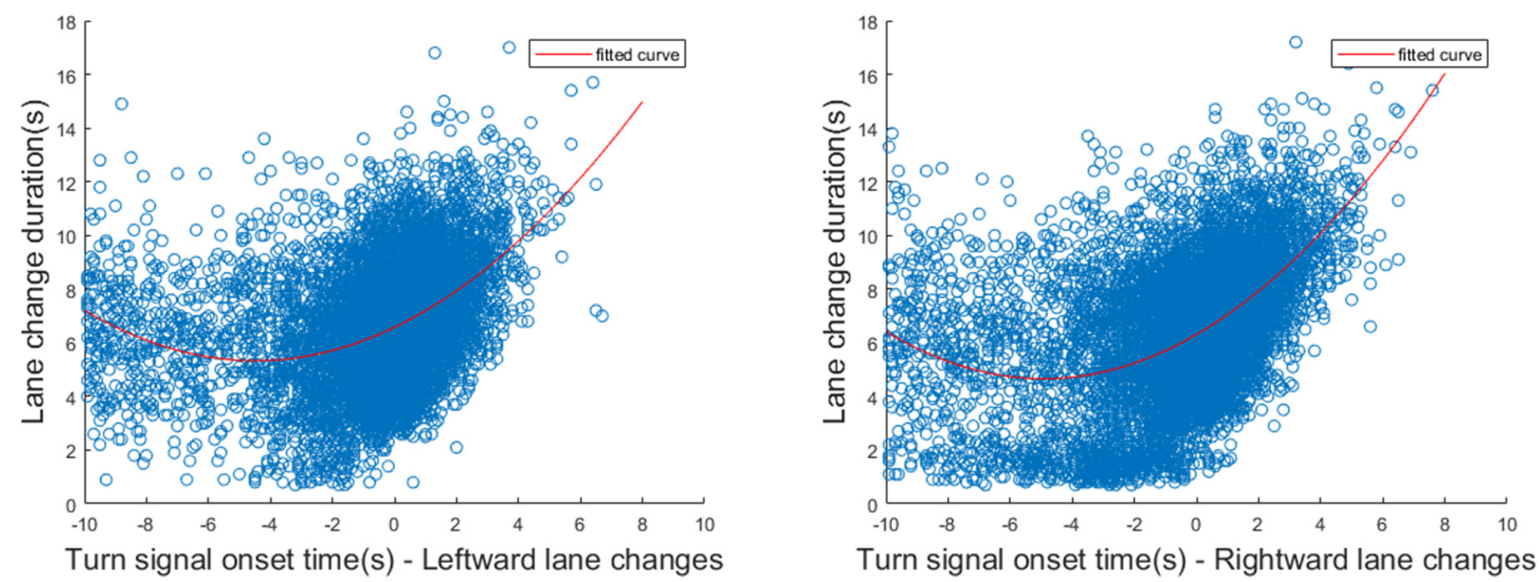

Figure 3. Plotted relationship between turn signal onset time and lane change duration

\section{CONCLUSION}

In summary, this study shows the basic features about overtaking behavior on freeways, including turn signal use, the distributions of turn signal onset time, and the relationship between the onset time of turn signal use and the lane change duration, for both leftward and rightward lane changes. There were about $70 \%$ of lane changes with turn signal activated, and drivers used the turn signal earlier for rightward lane changes than for the left. For US freeway, the rightward lane changes usually have more interactions with the other road users (Lee et al., 2004). Compared to leftward overtaking, the drivers would activate the turn signal earlier to provide more communication space with other vehicles.

In the naturalistic driving data extraction in this study, it is challenging to tell if the driver is overtaking a slower lead vehicle, or just discretionarily changing lanes with an existing lead vehicle, unless the driver's intention is clear and the route is known. One limitation of this study is that the overtaking events may indicate the other intention, such as the preparation of taking the exit ramps. Also, due to the limitation of the vision-based and Dedicated Short-Range Communication (DSRC) based sensors that were instrumented in the SPFOT vehicles, we do not have sufficient quantitative data for all the ambient traffic counts.

Finally, this study examined how the onset time of turn signal use is related to lane change duration. The convex-shaped contour shows that drivers will have longer lane change duration when they activate the turn signal too early (for complicated scenarios or by conservative drivers) or too late (minor lateral movement before applying the turn signal). As mentioned in the previous research, the lane change duration might be affected by traffic conditions and other vehicles around the host vehicle (Toledo \& Zohar, 2007). Although this study simplified the 
scenarios as overtaking a lead vehicle, more conditional variables should be included in the future work.

In conclusion, this study was designed to understand some features related to lane change maneuvers, which are critical for AV to predict and anticipate the other road users' maneuver intentions. As this study provides some basic examination related to lane change intention prediction, future analysis are needed including the consideration of other vehicles' locations and more lane change scenarios to develop more accurate and comprehensive prediction models for the AV to predict the other vehicles' moving trajectories.

\section{REFERENCES}

Bezzina, D., Sayer, J. (2015). Safety Pilot Model Deployment: Test Conductor Team Report (DOT HS 812 171). Retrieved from Washington, D.C.

Chen, D. Y., Peng, Y. J., Chen, L. C., \& Hsieh, J. W. (2014). Nighttime turn signal detection by scatter modeling and reflectance-based direction recognition. IEEE Sensors Journal, 14(7), 2317-2326.

Doshi, A. \& Trivedi, M. (2008). A comparative exploration of eye gaze and head motion cues for lane change intent prediction. Paper presented at IEEE Intelligent Vehicles Symposium, Eindhoven, The Netherlands.

Fröhlich, B., Enzweiler, M., \& Franke, U. (2014). Will this car change the lane? Turn signal recognition in the frequency domain. Paper presented at IEEE Intelligent Vehicles Symposium, Dearborn, MI, USA.

Kumar, P., Perrollaz, M., Lefèvre, S., \& Laugier, C. (2013). Learning-based approach for online lane change intention prediction. Paper presented at IEEE Intelligent Vehicles Symposium, Gold Coast, Australia.

Lee, S. E., Olsen, E. C. B., \& Wierwille, W. W. (2004). A comprehensive examination of naturalistic lane-changes (DOT HS 809 702). Retrieved from Washington, D.C.

Schmidt, K., Beggiato, M., Hoffmann, K. H., \& Krems. J. F. (2014). A mathematical model for predicting lane changes using the steering wheel angle. Journal of Safety Research, 49, 85-90.

Tijerina, L., \& Hetrick, S. (1997). Analytical evaluation of warning onset rules for lane change crash avoidance systems. Paper presented at the Human Factors and Ergonomics Society 41st Annual Meeting, Albuquerque, NM.

Toledo, T. \& Zohar, D. (2007). Modeling duration of lane changes. Transportation Research Record, 1999, 71-78.

Wang, M., Hoogendoorn, S. P., Daamen, W., van Arem, B., \& Happee, R. (2015). Game theoretic approach for predictive lane-changing and car-following control. Transportation Research Part C, 58(A), 73-92. 